\title{
Magnetic Resonance Imaging of Pulmonary Damage in the Term and Premature Rat Neonate Exposed to Hyperoxia
}

\author{
CHRISTOPHER J. APPLEBY AND RHEAL A. TOWNER \\ North Queensland Magnetic Resonance Centre, School Biomedical and Molecular Sciences, James Cook \\ University, Townsville, Queensland, Australia 4811
}

\begin{abstract}
ABST
Immaturity and oxygen toxicity have been implicated in the
pathogenesis of the neonatal disease bronchopulmonary dyspla-
sia. The present study aimed to investigate the use of magnetic
resonance imaging (MRI) to assess hyperoxia-mediated lung
injury in the term and premature neonate. Term (gestation, 22 d)
and premature ( 21 d) rat pups were exposed to hyperoxia
( $>95 \%)$ or air for a 6 -d period ( $n=7)$ and assessed for lung
damage by MRI. Pulmonary signal intensities of $\mathrm{T}_{1}$-weighted
images were significantly increased in both hyperoxia-exposed
term and premature neonates, relative to air-breathing controls $(p$
$<0.01)$. $\mathrm{T}_{2}$-weighted MRI signal intensities were also greater in
premature and term rat pups exposed to hyperoxia, but failed to
reach significance ( $p>0.05)$. Elevated MRI pulmonary signal
intensities may have represented an increase in magnetic reso-
nance-detectable free water, possibly indicating an increase in
edema. Corresponding histologic evidence of lung injury was
detected in both term and premature rat pups exposed to hyper-
oxia. Histologic samples indicated focal regions of alveolar
hemorrhage, immune cell infiltration, edema, and collapse in
both term and premature rat neonates exposed to hyperoxia.
Alveolar air space was assessed ( $n=5$ ) by light microscopy
within a 0.5 mm ${ }^{2}$ region of the superior left and inferior right
\end{abstract}
pulmonary lobes of each treatment group. Alveolar area of the superior left lung lobe of the premature hyperoxia treatment group was significantly smaller than other treatment groups $(p<$ $0.05)$. Reduced area for respiratory exchange was probably a result of observed focal areas of edema and collapse. MRIdetectable increases in lung signal intensity may have represented an increase in hyperoxia-induced pulmonary edema in the 6-d-old rat neonate. Increases in signal intensity correlated with the appearance of edema in pulmonary histologic samples. Premature delivery had a less defined effect on lung injury but possibly exacerbated hyperoxia-mediated pulmonary damage.

(Pediatr Res 50: 502-507, 2001)

Abbreviations
MRI, magnetic resonance imaging
MR, magnetic resonance
RDS, respiratory distress syndrome
BPD, bronchopulmonary dysplasia
TR, repetition time
TE, echo time
VS2D, verticle scale in two dimensions

Normal pulmonary growth and development are disrupted at premature birth. In the premature human neonate, ventilation with elevated oxygen concentrations is often required to correct hypoxemia at room air. Hyperoxia has been implicated in the development of both RDS and BPD (1). Pulmonary oxygen toxicity has been studied in a number of species (2-4) and tends to develop into two well-described stages, representing a transition from acute to chronic lung injury. The initial acute phase involves the accumulation of proteinaceous edema and the development of fibrin-rich membranes, whereas chronic injury is characterized by metaplasia, immune cell infiltration,

Received June 23, 2000; accepted April 27, 2001.

Correspondence and reprint requests: Rheal A. Towner, M.D., North Queensland Magnetic Resonance Centre, School of Biomedical and Molecular Sciences, James Cook University, Townsville, Queensland, Australia, 4811; e-mail: Rheal.Towner@jcu.edu.au Supported by a James Cook University Internal Research Grant. and fibrosis (5). Examples of hyperoxia-induced changes in pulmonary architecture have been documented in the adult (6), immature rat $(7,8)$, and the neonatal rat pup (9).

MRI is primarily based on the detection of a signal from the hydrogen nuclei of water owing to the magnetic properties of water molecules when exposed to a magnetic field. Therefore, an increase in hydrogen nuclei from free water will appear as an increase in image signal intensity in standard spin echo $\mathrm{T}_{1}$-weighted images. Hayes et al. (10) reported that regional pulmonary edema simulated by saline installation into the lungs of dead rats was detectable by MRI as an increase in signal intensity, which correlated well with gravimetric measurements of lung water. A number of studies have subsequently used MRI to assess differences in pulmonary oxygen toxicity caused by particular dietary deficiencies (11-13). Taylor et al. (14) used MRI to study the effect of glutathione 
concentration on oxygen susceptibility of weanling rats. Rats exposed to an $85 \%$ oxygen concentration for a $4-d$ period, and on a protein-deficient diet lacking a glutathione supplement, exhibited pulmonary edema, which appeared as an increase in lung image signal intensity.

The purpose of this study was to investigate the effects of a 6-d exposure to $>95 \%$ oxygen on the lungs of term and premature rat pups and to study the value of MRI as a possible clinical tool for the noninvasive assessment of pulmonary damage in the neonate.

\section{METHODS}

Paired breeding. Sixteen, female Sprague Dawley rats (250-300 g) from the James Cook University breeding colony were numbered and divided into four groups, and each group was placed with one male overnight. The presence of sperm in vaginal smears taken the following morning and stained with methylene blue (Difco Laboratories, Detroit, MI, U.S.A.) indicated successful conception at time 0 . Identified pregnant females were then segregated for term delivery $(22 \mathrm{~d}$ gestation). After a one-night interval, a further group of 16 females were introduced to males overnight. Those females identified as pregnant from vaginal smears the next day were separated for premature delivery $(21 \mathrm{~d})$.

Hyperoxia and normoxia exposures. Term and premature rat pups together with a suckling mother were placed within cages into a Plexiglas chamber $(51 \times 36 \times 31 \mathrm{~cm})$ with an inlet and outlet port for oxygen or air. Flow rate was maintained at $8.0 \mathrm{~L} / \mathrm{min}$, and $\mathrm{O}_{2}$ concentration $(>95 \%)$ was monitored continuously (oxygen analyzer, Teledyne Electronic Devices, Los Angeles, CA, U.S.A.). Room temperature was maintained at $24-25^{\circ} \mathrm{C}$ on a 12 -h on/12-h off light cycle. Food (standard rat chow) and water was available to the mothers ad libitum. Rat pups were exposed to normoxia or hyperoxia for a 6-d period. Ethics approval was obtained from the Experimentation Ethics Review Committee of James Cook University (ethics approval number A474).

Term delivery. Paired, timed pregnant Sprague Dawley rats were separated for term delivery ( $22 \mathrm{~d}$ ). One litter was exclusively exposed to hyperoxia and the other exposed to normoxia. Mothers were alternated between the two rat pup treatment groups every $24 \mathrm{~h}$ to prevent previously documented adult rat oxygen toxicity (15) and any inconsistencies in pup nutrition.

Premature delivery. Two timed pregnant females were euthanized at $21 \mathrm{~d}$ of gestation by cervical dislocation, and the rat pups were delivered immediately by hysterotomy. Adult females were killed at $\mathrm{d} 21$ by cervical dislocation, as anesthesia results in fetal depression and rat pups fail to survive delivery $>24 \mathrm{~h}$ before term (16). After pup delivery the airway was cleared of fluid with paper towel, and the abdomen was stimulated with two fingers, moving the chest in an up and down motion until breathing was initiated by a sharp intake of breath. The umbilical cord was then cut just below the placenta, and any blood in the cord was removed by squeezing with a pair of forceps to delay more profuse cord bleeding. Rat pups were then washed in tap water $\left(28^{\circ} \mathrm{C}\right)$ and dried. The umbilical cord was tied with a piece of cotton thread at the abdomen, and the remaining cord cut above the ligature. Rat pups were washed again thoroughly to ensure all traces of the mother's blood had been removed to prevent cannibalism by the surrogate (16). Rat pups were then placed on a heated mat and separated into two groups of no more than 15 individuals. After $30 \mathrm{~min}$ each group of rat pups was introduced to a surrogate mother bred $48 \mathrm{~h}$ before those required for hysterotomy. One mother and litter were exposed to hyperoxia while the other group was exposed to normoxia for a 6-d period. During exposure surrogate mothers were alternated between treatment groups every $24 \mathrm{~h}$ to prevent oxygen toxicity (15). At d 6, rat pup weight was recorded.

Magnetic resonance imaging. Term and premature rat pups were imaged immediately after $6 \mathrm{~d}$ of hyperoxic or normoxic exposure $(n=7)$. Rat pups were anesthetized with $1.5 \%$ isoflurane (Lyppard, Lyppard Queensland Pty Ltd, Townsville, Qld, Australia) in oxygen $\left(>95 \% \mathrm{O}_{2}\right)$ at a flow rate of 0.5 $\mathrm{L} / \mathrm{min}$. Rat pups were then placed into a ${ }^{1} \mathrm{H}-\mathrm{MR}$ birdcage probe and imaged (TR, $0.80 \mathrm{~s}$; TE, $0.024 \mathrm{~s}$; Varian INOVA UNITY 7.0-T, 18-cm horizontal-bore imaging spectrometer). Each image (transverse orientation) was $2 \mathrm{~mm}$ thick with a $128 \times 128$ matrix and four averages per acquisition. $\mathrm{T}_{1}(\mathrm{TR}, 0.80 \mathrm{~s}$; TE, $0.024 \mathrm{~s}$ ) and $\mathrm{T}_{2}$ (TR, $2.0 \mathrm{~s}$; TE, $0.06 \mathrm{~s}$ ) -weighted imaging was performed sequentially on each experimental animal.

Image analysis. MR image signal intensity was standardized $\left(T_{1} V S 2 D=4250 ; T_{2} V S 2 D=6000\right)$. A $2-\mathrm{mm}^{2}$ region of the top, middle, and lower portion of the chest cavity was analyzed for signal intensity (ImagePro Plus, Media Cybernetic, Silver Springs, MD, U.S.A.) from which an average was calculated. The left portion of the chest cavity was analyzed to minimize the effect of cardiac motion. Signal intensity data from the lungs of term and premature rat pups exposed to hyperoxia and normoxia $(n=7)$ were analyzed using the Wilcoxon's rank sum test for nonparametric data (Statistica, Statsoft, Tulsa, OK, U.S.A.).

Histology. Term and premature rat pups exposed to hyperoxia and normoxia for $6 \mathrm{~d}$ were anesthetized with $1.5 \%$ isoflurane at a flow rate of $0.5 \mathrm{~L} / \mathrm{min}$ (Lyppards). A piece of string was tied tightly around the neck until the trachea was completely constricted. The chest cavity was then carefully opened and the abdomen removed. The head and thorax, with the ligature still in place around the neck, were placed into $10 \%$ formalin ( $10 \%$ formaldehyde in water) and refrigerated. After $7 \mathrm{~d}$ the lungs were removed and placed into fresh formalin. The left superior and right inferior lobes of the lung were selected

Table 1. Body weight at $6 d$ as a function of treatment

\begin{tabular}{cc}
\hline Treatment group & Body weight \\
\hline TA & $15.4 \pm 0.63$ \\
TH & $14.10 \pm 0.96^{*}$ \\
PA & $15.04 \pm 0.65$ \\
PH & $13.10 \pm 3.50 \dagger$ \\
\hline
\end{tabular}

Values are mean $\pm \mathrm{SD}(n=15)$.

Abbreviations: TA, term air; TH, term hyperoxia; PA, premature air; PH, premature hyperoxia.

$* p=0.01$, TA $v s$ TH.

$\dagger p<0.01$, PA vs PH. 


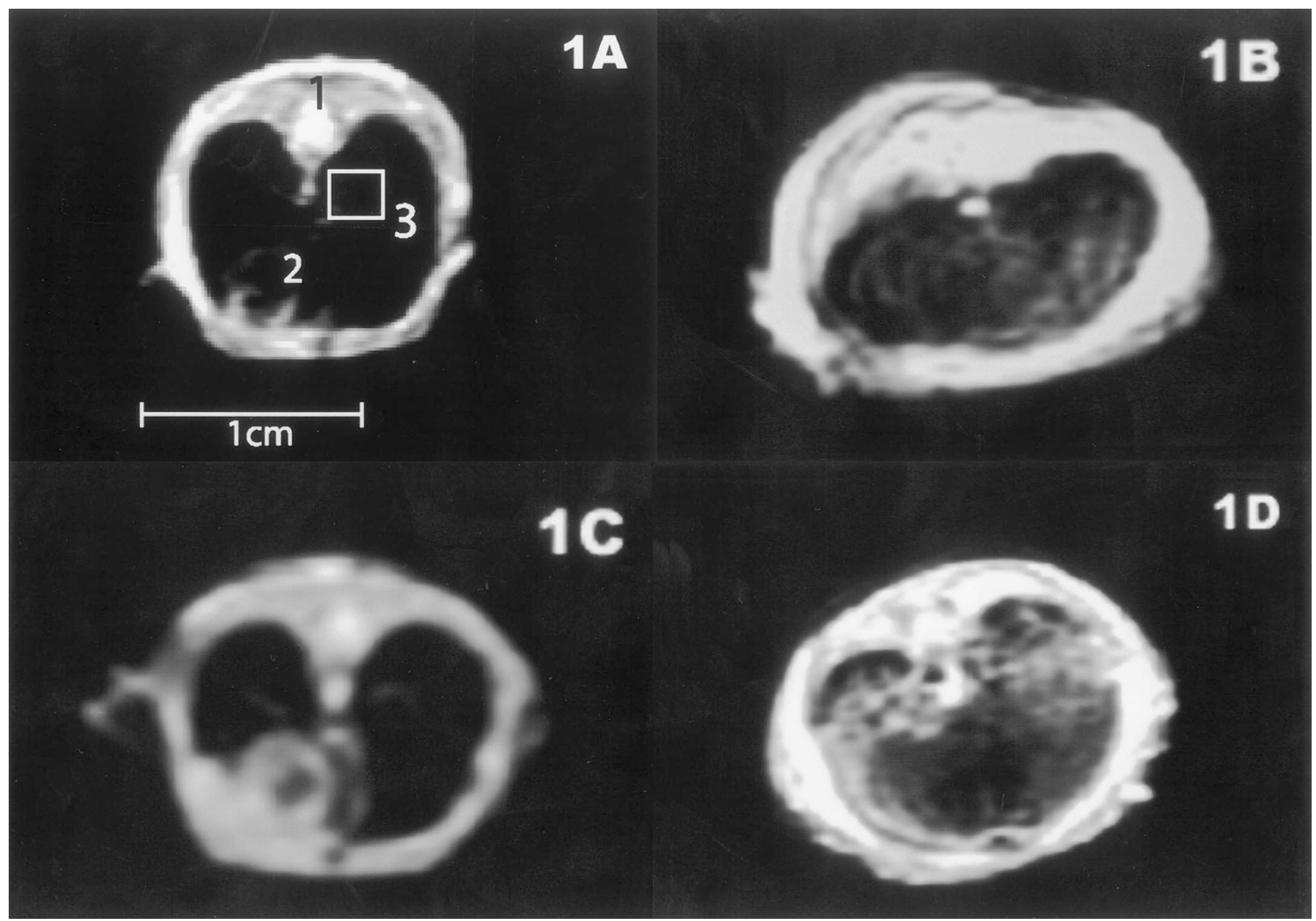

Figure 1. $\mathrm{T}_{1}$-weighted MR images of pulmonary transverse sections of term and premature rat neonates exposed to normoxia and hyperoxia for a 6-d period. $A$, term air. Assignments: (1) spinal cord, (2) heart, (3) upper right region, analyzed for signal intensity. $B$, term hyperoxia. $C$, premature air. $D$, premature hyperoxia.

for sectioning as these were the two largest lobes of $\mathrm{d} 6$ rat pups after dehydration (unpublished data). Lung tissue was dehydrated in graded ethanols, cleared in xylene, and embedded in paraffin wax. Sections $(5 \mu \mathrm{m})$ were cut using a Jung model 1130 rotary microtome (Selby's Scientific, Brisbane, QLD), stained with hematoxylin and eosin, and mounted on slides in DePeX (DePeX, Serva, Heidelberg, Germany). Photomicrographs were obtained using a Leitz Vario-orthoplan photomicroscope (Gladesville, NSW, Australia).

Histologic analysis. An image from a Leica microscope $(\times 10$ magnification) was captured via computer program (Spot Diagnostics Inc, Brisbane, Australia) from the left superior and right inferior pulmonary lobe of each treatment group. Images were transferred to an image analysis program (ImagePro Plus, Media Cybernetic). Section field of view was calibrated from the captured image (Leica microscope, $\times 10$ magnification, Gladesville, NSW, Australia) of a 1-mm rule slide (Olympus, Tokyo, Japan). A scale was placed on each section image, and total alveolar air space was calculated for a $0.5-\mathrm{mm}^{2}$ region from the same scale coordinates on each slide section. Total alveolar area was obtained from the sum multiplications of the measured $x$ and $y$ lengths of individual alveolar air spaces within the $0.5-\mathrm{mm}^{2}$ defined region of each slide. Alveolar air space area was calculated for each lobe of each treatment group and then analyzed $(n=5)$ by two-tailed $t$ test for parametric data (Statistica, Statsoft).

\section{RESULTS}

Growth. Term air neonates gained the most weight at d 6, followed by the premature normoxia treatment group (Table 1). Hyperoxia appeared to result in a significant reduction in body weight in both term hyperoxia $(p=0.01)$ and premature hyperoxia $(p>0.01)$ treatment groups relative to respective air-breathing controls. No significant difference was found to occur between term and premature hyperoxia, or term and premature normoxia, rat pup weights at $\mathrm{d} 6$.

MRI. $\mathrm{T}_{1}$-weighted MR images of term and premature rat pups exposed to hyperoxia indicated a greater pulmonary signal intensity than those of premature and term neonates exposed to room air (Fig. 1). Mean image signal intensity taken from $2-\mathrm{mm}^{2}$ regions of the left portion of the chest cavity was significantly higher in both term $(p<0.01 ; n=7)$ and premature $(p=0.01 ; n=7)$ rat pups exposed to hyperoxia, relative to air-breathing controls (Fig. 2). Mean image signal intensity was the highest in the premature hyperoxia group but 


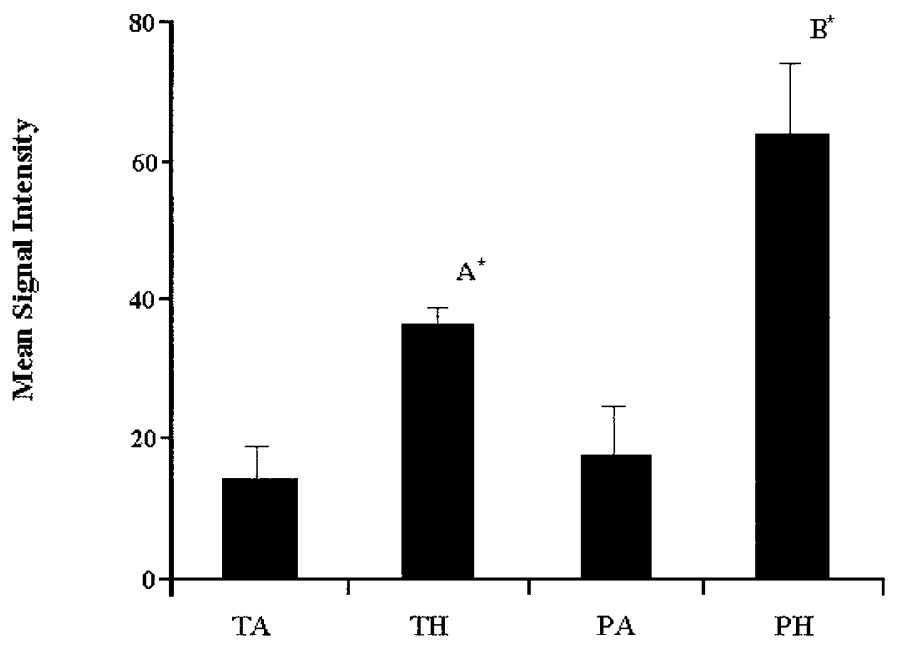

Treatment Group

Figure 2. Mean $\mathrm{T}_{1}$-weighted image signal intensity and $\mathrm{SE}(n=7)$ of $2-\mathrm{mm}^{2}$ regions taken from the upper, middle, and lower left portion the chest cavity of term and premature rat neonates exposed to normoxia and hyperoxia for a 6-d period. $T A$, term air; $T H$, term hyperoxia; $P A$, premature air; $P H$, premature hyperoxia. $\mathrm{A}^{*}, p<0.01$, TA $v s \mathrm{TH} ; \mathrm{B}^{*}, p=0.01 \mathrm{PA} v s \mathrm{PH}$.

was not significantly different from hyperoxia-exposed term rat pups. No significant difference was found between premature and term air-breathing controls. $\mathrm{T}_{2}$-weighted images taken immediately after $\mathrm{T}_{1}$ MRI recorded similar, but not significant, differences in signal intensity data among treatment groups (Fig. 3).

Histology. Photomicrographs of both term (Fig. 4B) and premature (Fig. 4D) hyperoxia-exposed rat pups displayed focal losses of pulmonary architecture within individual pulmonary lobes. A decrease in the number and size of alveoli in hyperoxia-treated groups appeared to correlate with an increase

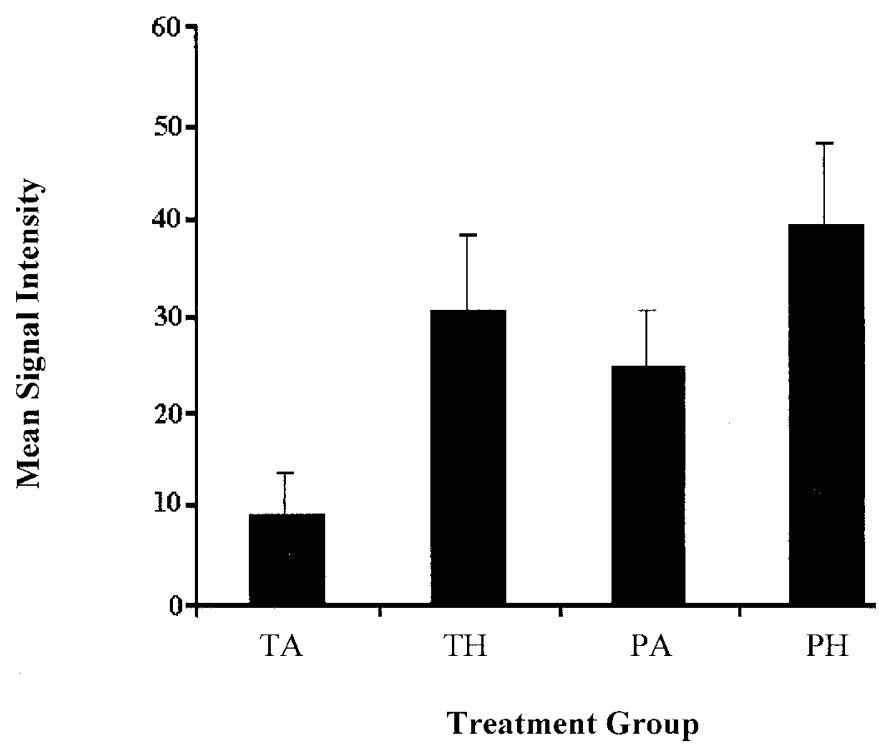

Figure 3. Mean $\mathrm{T}_{2}$-weighted image signal intensity and $\mathrm{SE}(n=7)$ of $2-\mathrm{mm}^{2}$ regions taken from the upper, middle, and lower left portion of the chest cavity of term and premature rat neonates exposed to normoxia and hyperoxia for a 6-d period. $T A$, term air; $T H$, term hyperoxia; $P A$, premature air; $P H$, premature hyperoxia. in airway thickness. There was also evidence of pulmonary edema, immune cell infiltration, and hemorrhage.

Analysis of superior left lung lobe mean alveolar air space size revealed significantly lower air space size in the premature hyperoxia treatment group relative to all other groups (Fig. 5). Sections taken from premature air-breathing controls appeared to be composed of larger alveoli than the term air group, although this difference failed to reach significance. Term hyperoxia mean alveolar air space size was smaller than the term normoxia group but did not differ significantly. Analysis of inferior right lung lobe mean alveolar air space size produced smaller, insignificant differences in alveolar air space size (data not included).

\section{DISCUSSION}

Hyperoxia-induced pulmonary injury is thought to occur because of several different contributing factors, including free radical generation, a result of oxidant insult and release from activated immune cells, and a compromised antioxidant enzyme system. Degree of neonatal prematurity may also play an important role in determining the level of pulmonary damage. Premature neonates in a number of different species have been shown to increase pulmonary antioxidant enzymes rapidly in the last 10 to $15 \%$ of gestation (17), whereas pulmonary surfactant maturation has been shown to occur late in human gestation (18). Therefore, premature neonates, in which pulmonary development has been disrupted, may be more vulnerable to hyperoxia-induced pulmonary damage. The 21-d rat pup has been previously reported as biochemically immature, with lower pulmonary antioxidant (19) and surfactant levels (17) relative to the term neonate. The 1-d premature rat pup may therefore be regarded as a viable model to investigate the effects of both hyperoxia and premature delivery on pulmonary development.

The accumulation of proteinaceous pulmonary edema occurs during both acute and chronic hyperoxia-induced lung injury (5). After 6-d hyperoxia exposure $\mathrm{T}_{1}$-weighted MR images of both term and premature rat neonates indicated a significant increase in lung signal intensity. The ability of MRI to detect soft tissue damage in vivo is associated with the $\mathrm{T}_{1}$ (spin lattice or longitudinal TR) and $\mathrm{T}_{2}$ (spin-spin or transverse TR) properties of the hydrogen in water. These properties modulate tissue signal intensity and are dependent on tissue composition. Generally, free or unbound water is characterized by a prolonged $T_{1}$ and $T_{2} T R$ relative to nondiseased tissue. Diseased tissue often has increased intracellular water, possibly owing to edema and cellular necrosis, which may contribute to longer $T_{1}$ and $T_{2}$. Prolonged $T_{1}$ and $T_{2}$ values of diseased tissue set against the intrinsically short $\mathrm{T}_{1}$ and $\mathrm{T}_{2}$ of surrounding normal tissue creates a detectable contrast. Pulmonary damage as detected by MRI may be particularly obvious when compared with the low water content of the nondiseased aerated lung. MR image signal intensity in the lungs has previously been shown to correlate well with pulmonary water content. Cutillo et al. (20) discovered that MR image signal intensity was within $20 \%$ agreement of conventional gravimetric measurements of excised, unperfused adult rat lung. 


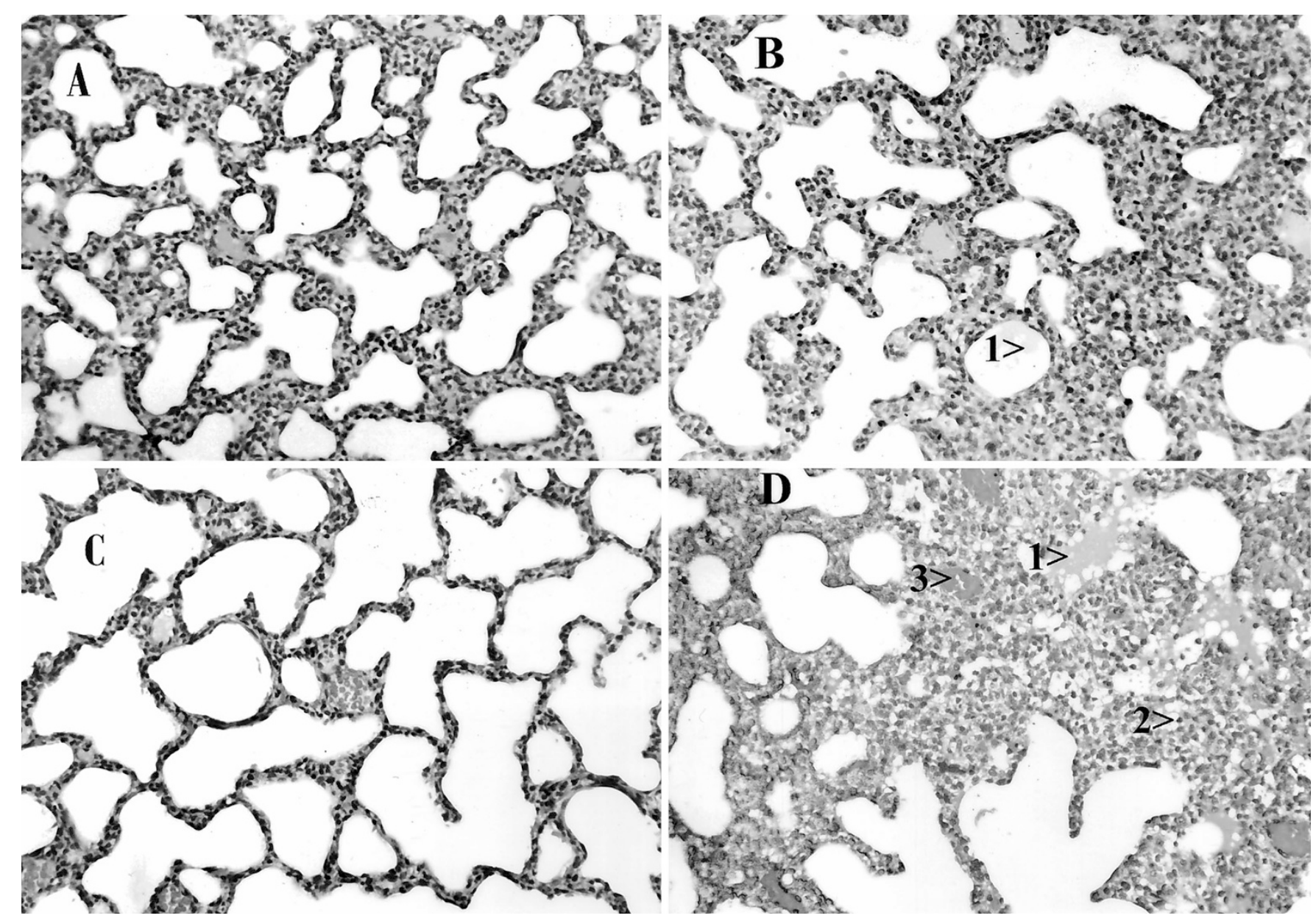

Figure 4. Histologic evidence of pulmonary edema in the hyperoxia-exposed rat neonate treatment groups (hematoxylin and eosin, original magnification $\times 51.2$ ). A, term air. $B$, term hyperoxia. $C$, premature air. $D$, premature hyperoxia. Assignments: (1) intraalveolar edema, (2) immune cell infiltration, (3) blood vessel congestion.

Therefore, the observed significant increase in pulmonary signal intensity in the premature and term rat neonate exposed to hyperoxia, relative to air-breathing controls, may represent a significant increase in lung water content. Cutillo et al. (21) produced a similar increase in MR image lung signal intensity in an oleic acid-induced model of pulmonary edema in the adult rat. Lung signal intensity was relatively higher in premature rat neonates exposed to hyperoxia than in term pups, but this difference was not significant. This may have been partly because of the high degree of variability in signal intensities within treatment groups, which may have resulted from individual differences in resistance to hyperoxia. Histologic evidence also indicated that the degree of lung damage varied within treatment groups. Differences in $\mathrm{T}_{2^{-}}$ weighted MR image signal intensities were similar to those recorded for $T_{1}$ data. However, recorded differences in $T_{2}$ data among treatment groups was not significantly different. This may have been caused by a poor signal to noise ratio, as respiratory gating was not implemented because of shallow breathing of anesthetized pups. Wexler et al. (22) discovered a close correlation existed between $T_{1}$ TRs and lung water content in a saline lavage-induced canine model of pulmonary edema. However, an accurate determination of $\mathrm{T}_{2}$ TRs was not possible owing to poor signal to noise, a result of motion artifacts as respiratory gating was not used.
Observed increases in MR image pulmonary signal intensities correlated with the appearance of edema in lung sections. Histologic evidence of pulmonary injury also included immune cell infiltration and hemorrhage. Bucher et al. (23) reported that newborn and 1-wk-old rat pups exposed to $80 \% \mathrm{O}_{2}$ for a 6-d period exhibited interstitial edema and congestion. Sections taken from the lungs of hyperoxia-treated rat pups appeared to have fewer, smaller alveoli. This was in agreement with a previous study that found neonatal oxygen exposure resulted in a decrease in the number of alveoli per square millimeter (24). Mean alveoli area was significantly smaller in premature hyperoxia-exposed neonates relative to all other treatment groups including the term hyperoxia group. A reduction in mean alveolar area may have been related to an observed decrease in alveolar size resulting from a loss of architecture and alveolar air space in filling because of hemorrhage and edema (25). A decrease in respiratory area as a result of hyperoxia has been reported previously in the term rat neonate exposed to $>95 \% \mathrm{O}_{2}$ for $7 \mathrm{~d}$ (8). Differences in respiratory exchange area were not significant in the right inferior pulmonary lobe, emphasizing the focal nature of lung damage induced after 6-d hyperoxia exposure.

It appears that MRI of the rat neonate may be used to investigate the effects of hyperoxia-mediated lung injury. MRI 


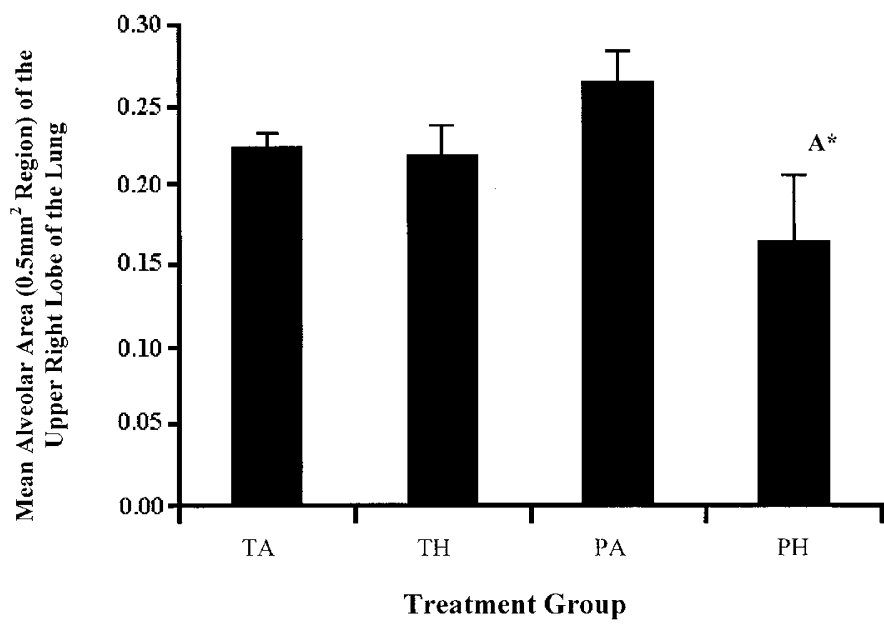

Figure 5. Mean total alveolar area and SE $(n=5)$ within a $0.5-\mathrm{mm}^{2}$ region of the superior left pulmonary lobe of term and premature rat neonates exposed to normoxia and hyperoxia. $T A$, term air; $T H$, term hyperoxia; $P A$, premature air; $P H$, premature hyperoxia. $\mathrm{A}^{*}, p<0.01$, $\mathrm{PH} v s \mathrm{PA} ; p<0.05$, $\mathrm{PH} v s \mathrm{TH}$ and $\mathrm{PH} v s \mathrm{TA}$

data indicated the presence of edema, represented by a quantifiable increase in lung signal intensity, which corroborated with pulmonary histology. It appears that premature delivery did have an effect on pulmonary development, but these results proved less conclusive. MRI signal intensity was greater in the premature neonate relative to the term hyperoxia treatment group in both $\mathrm{T}_{1}$ - and $\mathrm{T}_{2}$-weighted imaging, but failed to reach significance. However, a significant reduction in respiratory exchange area was recorded within the superior left pulmonary lobe of the premature hyperoxia group, relative to all other treatment groups, including the term hyperoxia neonate. No significant difference was found to occur between the weights of d 6 premature and term neonates exposed to hyperoxia. However, term and premature neonates exposed to normoxia were significantly heavier than hyperoxia-breathing counterparts, suggesting slower growth and development rates in the hyperoxia-exposed neonate. Therefore, the effects of hyperoxia may have had a more significant effect on lung development in the 6-d-old neonate than did 1-d premature delivery. MRI after longer periods of hyperoxia exposure at different times points may further clarify the effect of premature delivery on lung development. However, these results indicate that prematurity may have to some degree exacerbated observed hyperoxiainduced lung injury.

Acknowledgments The authors thank Laurie Reilly (Department of Physiology and Pharmacology) for advice and assistance in the preparation of histology and Prof. Phillip Summers
(Department of Physiology and Pharmacology) for advice on subsequent pathologic diagnosis. We also thank Dr. Linda Crane and Scott Blyth (Department of Physiology and Pharmacology) for advice on rat reproduction.

\section{REFERENCES}

1. O’Brodovich HM, Mellins RB 1985 Bronchopulmonary dysplasia. Unresolved neonatal acute lung injury. Am Rev Respir Dis 132:694-709

2. Frank L, Sosenko RS 1991 Failure of premature rabbits to increase antioxidant enzymes during hyperoxic exposure: increased susceptibility to pulmonary oxygen toxicity compared with term rabbits. Pediatr Res 29:292-296

3. Delemos RA, Coalson JJ, Gerstmann DR, Kuehl TJ, Null DM 1987 Oxygen toxicity in the premature baboon with hyaline membrane disease. Am Rev Respir Dis 136:677-682

4. Warner BB, Stuart LA, Papes RA, Wispe JR 1998 Functional and pathological effects of prolonged hyperoxia in neonatal mice. Am J Physiol 275:L110-L117

5. Small A 1984 New perspectives on hyperoxic pulmonary oxygen toxicity-A review. Undersea Biomed Res 11:1-24

6. Crapo JD, Barry BE, Foscue HA, Shelbourne J 1980 Structural and biochemical changes in rat lungs occurring during exposure to lethal and adaptive doses of oxygen. Am Rev Respir Dis 122:123-143

7. Solway J, Hershman MB 1995 Structural and functional abnormalities of the airways of hyperoxia-exposed immature rats. Chest 107(suppl):89S-93S

8. Harrison G, Rosan RC, Sloane A 1970 Bronchiolitis induced by experimental acute and chronic oxygen intoxication in young adult rats. J Pathol 102:115-122

9. Shaffer SG, O'Neill D, Brandt SK, Thibeault DW 1987 Chronic vascular pulmonary dysplasia associated with neonatal hyperoxia exposure in the rat. Pediatr Res 21:14-20

10. Hayes CE, Case TA, Ailion DC, Morris AH, Cutillo A, Blackburn CW, Durney CH, Johnson SA 1982 Lung water quantitation by nuclear magnetic resonance imaging. Science 216:1313-1315

11. Taylor CG, McCutchen TL, Boermans HJ, DiSilvestro RA, Bray TM 1997 Comparison of $\mathrm{Zn}$ and vitamin $\mathrm{E}$ for protection against hyperoxia-induced lung damage. Free Radic Biol Med 22:543-550

12. Taylor CG, Bray TM 1991 Increased lung copper-zinc-superoxide dismutase activity and absence of magnetic resonance imaging-detectable lung damage in copperdeficient rats exposed to hyperoxia. J Nutr 121:467-473

13. Taylor CG, Towner RA, Janzen EG, Bray TM 1990 MRI detection of hyperoxiainduced lung edema in Zn-deficient rats. Free Radic Biol Med 9:229-233

14. Taylor CG, Bauman PF, Sikorski B, Bray TM 1992 Elevation of lung glutathione by oral supplementation of L-2-oxothiazolidine-4-carboxylate protects against oxygen toxicity in protein-energy malnourished rats. FASEB J 6:3101-3107

15. Tanswell AK, Freeman BA 1987 Liposome-entrapped antioxidant enzyme prevent lethal $\mathrm{O}_{2}$ toxicity in the newborn rat. J Appl Physiol 63:347-352

16. Tanswell AK, Wong L, Possmayer F, Freeman BA 1989 The preterm rat: a model for studies of acute and chronic neonatal lung disease. Pediatr Res 25:525-529

17. Frank L, Sosenko RS 1987 Prenatal development of lung antioxidant enzymes in four species. J Pediatr 10:106-110

18. Ikegami M, Jobe AH 1993 Surfactant metabolism. Semin Perinatol 17:275-284

19. Tanswell AK, Freeman BA 1984 Pulmonary antioxidant enzyme maturation in the fetal and neonatal rat. I. Developmental profiles. Pediatr Res 18:584-587

20. Cutillo AG, Morris AH, Blatter DD, Case TA, Ailion DC, Durney CH, Johnson SA 1984 Determination of lung water content and distribution by nuclear magnetic resonance. J Appl Physiol 57:583-588

21. Cutillo A, Goodrich KC, Ganesan K, Watanabe S, Ailion DC, Albertine KH, Morris AH, Durney CH 1995 Lung water measurements by nuclear magnetic resonance: correlation with morphometry. J Appl Physiol 79:2163-2168

22. Wexler RH, Nicholson RL, Prato FS, Carey LS, Vinitski S, Reese L 1985 Quantitation of lung water by nuclear magnetic resonance imaging. Invest Radiol 20:583590

23. Bucher JR, Roberts RJ 1981 The development of the newborn rat lung in hyperoxia: a dose-response study of lung growth, maturation, and changes in antioxidant enzyme activities. Pediatr Res 15:999-1008

24. Thibeault DW, Mabry S, Rezaiekhaligh M 1990 Neonatal pulmonary oxygen toxicity in the rat and lung changes with aging. Pediatr Pulmonol 9:96-108

25. Wilson WL, Mullen M, Olley PM, Rabinovitch M 1985 Hyperoxia-induced pulmonary vascular and lung abnormalities in young rats and potential for recovery. Pediatr Res 19:1059-1067 